\title{
Branching Patterns and Anatomical Course of the Common Fibular Nerve
}

\author{
Goo Young Kim, $\mathrm{MD}^{1}$, Chae Hyeon Ryou, $\mathrm{MD}^{1}$, Ki Hoon Kim, MD, PhD', \\ Dasom Kim, BS ${ }^{2}$ Im Joo Rhyu, MD, $\mathrm{PhD}^{2}$, Dong Hwee Kim, MD, $\mathrm{PhD}^{1}$ \\ ${ }^{1}$ Department of Physical Medicine and Rehabilitation, Korea University College of Medicine, Ansan; \\ ${ }^{2}$ Practical Anatomy Research Institute, Korea University College of Medicine, Ansan, Korea
}

Objective To present the branching patterns and anatomical course of the common fibular nerve (CFN) and its relationship with fibular head (FH).

Methods A total of 21 limbs from 12 fresh cadavers were dissected. The FH width (FH_width), distance between the FH and CFN (FH_CFN), and thickness of the nerve were measured. The ratio of the FH_CFN to FH_width was calculated as follows: $<1$, cross type and $\geq 1$, posterior type. Angle between the CFN and vertical line of the lower limb $5 \mathrm{~cm}$ proximal to the tip of the FH was measured. Branching patterns of the lateral cutaneous nerve of the calf (LCNC) were classified into four types according to its origin and direction as follows: type 1a, lateral margin of the CFN; type 1b, medial margin of the CFN; type 2, lateral sural cutaneous nerve (LSCN); and type 3, CFN and LSCN. Results In the cross type (15 cases, $71.4 \%$ ), the ratio of FH_CFN/FH_width was 0.83 and the angle was $13.0^{\circ}$. In the posterior type ( 6 cases, $28.6 \%$ ), the ratio was 1.04 and the angle was $11.0^{\circ}$. In the branching patterns of LCNC, type 2 was the most common (10 cases), followed by types $1 \mathrm{a}$ and $1 \mathrm{~b}$ (both, 5 cases).

Conclusion Location of the CFN around the FH might be related to the development of its neuropathy, especially in the cross type of CFN. The LCNC showed various branching patterns and direction, which could be associated with difficulties of electrophysiologic testing.

Keywords Peroneal nerve, Common fibular neuropathy, Cadaver, Fibula, Anatomy

\section{INTRODUCTION}

The common fibular nerve (CFN) is divided with the tibial nerve-a branch of the sciatic nerve-and is located in the lateral side compared to the tibial nerve. The CFN courses down from the medial side of the biceps

Received May 21, 2019; Accepted July 12, 2019

Corresponding author: Dong Hwee Kim

Department of Physical Medicine and Rehabilitation, Korea University College of Medicine, 123 Jeokgeum-ro, Danwon-gu, Ansan 15355, Korea. Tel: +82-31-412-5330, Fax: +82-31-412-5344, E-mail: rmkdh@korea.ac.kr

ORCID: Goo Young Kim (http://orcid.org/0000-0001-7698-2045); Chae Hyeon Ryou (http://orcid.org/0000-0003-3115-6465); Ki Hoon Kim (http:// orcid.org/0000-0002-7257-3858); Dasom Kim (http://orcid.org/0000-0002-7817-3652); Im Joo Rhyu (http://orcid.org/0000-0002-5558-6278); Dong Hwee Kim (http://orcid.org/0000-0002-8116-0078).

(c) This is an open-access article distributed under the terms of the Creative Commons Attribution Non-Commercial License (http://creativecommons.org/ licenses/by-nc/4.0) which permits unrestricted noncommercial use, distribution, and reproduction in any medium, provided the original work is properly cited. Copyright $\odot 2019$ by Korean Academy of Rehabilitation Medicine 
femoris tendon in the popliteal fossa and progresses to wrap the fibular head (FH). Further, it distributes the articular branch right after and eventually is separated into the superficial and deep fibular nerves [1].

Traumas such as bone fracture around the knee, severe ankle inversion, and direct nerve trauma cause CFN injury. However, compressive etiology is commonly observed [2]. Many bedridden patients in the intensive care unit have common fibular neuropathy due to nerve compression because of the position that the hip joint is externally rotated and the knee joint is flexed while in the supine state. Gravity, the shape of the lower extremity, and malnutrition contribute to the compression of the CFN, and pneumatic compression-a technique used to prevent deep venous thrombosis-is also believed to cause the compression of the CFN [3]. However, even in a similar environment, each individual differs in terms of nerve injury; thus, anatomy of the CFN around the FH must be assessed.

According to a case report by Khalil et al. [4], the lateral cutaneous nerve of the calf (LCNC) is the nerve responsible for lateral sensory in the proximal lower leg and popliteal fossa. In addition, the symptom of damage to the LCNC is similar to that of L5 radiculopathy; thus, examiners usually consider the latter, which is more likely to occur in patients presenting such symptom as tingling sense and hypoesthesia in the lower leg (predominantly in the lateral side). The LCNC is usually divided from the lateral sural cutaneous nerve (LSCN) - the branch of the CFN-and courses down in the lateral side (Fig. 1). However, the branching pattern of this nerve is extremely diverse and difficult to explain in detail.

This study aimed to assess the branching patterns of the CFN, including the LSCN and LCNC, and the relationship between the CFN and FH.

\section{MATERIALS AND METHODS}

A total of 21 limbs from 12 fresh cadavers were dissected ( 7 men, 5 women). The median age of cadavers was 79 years (range, 65-89 years). While in the prone position, the skin and subcutaneous tissue of the cadavers were dissected from the mid-thigh to the mid-leg and the course of the CFN around the FH and its branching patterns were examined. Because the LSCN, medial sural cutaneous nerve, and LCNC are extremely thin and su-

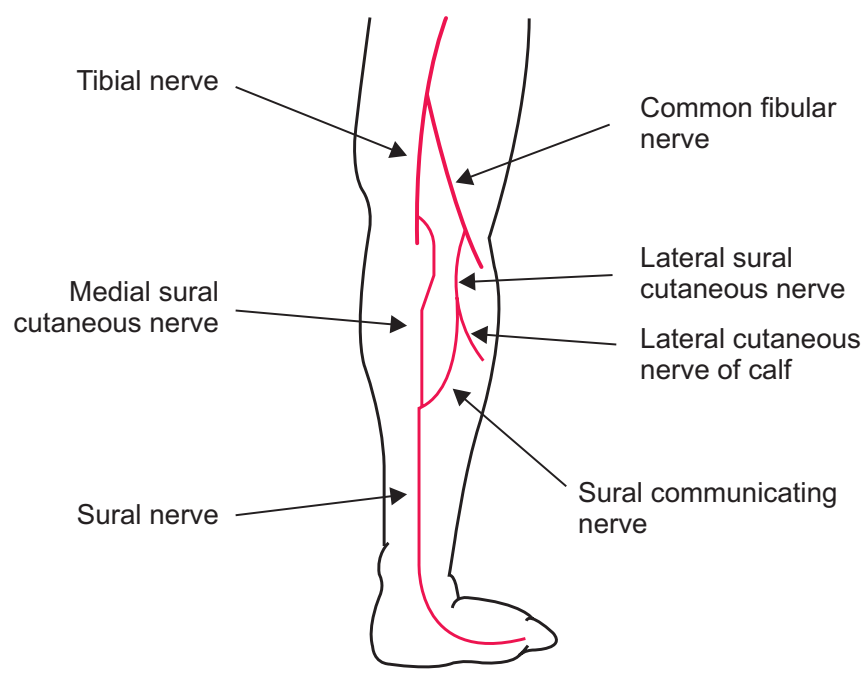

Fig. 1. Lateral cutaneous nerve of the calf.

perficially located, their dissection was difficult and complicated. The Institutional Review Board has confirmed that this cadaveric study is not subject to review.

On the mid-FH level, the width of the FH (FH_width) and distance between the anterior margin of the $\mathrm{FH}$ and CFN (FH_CFN) were measured using the Vernier caliper (Model S-19515; Shinwa Rules Co. Ltd., Sanjo, Japan) (Fig. 2). The course of the CFN around the FH was classified into two types: the cross type in which the CFN passes over the FH, and the posterior type in which the CFN is located posterior to the FH on the mid-FH level. If the ratio of the FH_CFN to FH_width was $<1$, it was considered the cross type, whereas if it was $\geq 1$, it was considered the posterior type.

The angle of the CFN to the vertical line of the lower limb $5 \mathrm{~cm}$ proximal to the tip of the $\mathrm{FH}$ was measured using a protractor.

The Mann-Whitney U test was used to assess whether there was a significant difference between the cross type and posterior type for the age, FH_CFN, FH_width, ratio (FH_CFN /FH_width), and angle.

Needle marking was done at the popliteal fossa (superior margin) and at $7 \mathrm{~cm}, 5 \mathrm{~cm}$, and $2 \mathrm{~cm}$ proximal to the tip of the $\mathrm{FH}$, midpoint of the $\mathrm{FH}$, and fibular neck, respectively. Subsequently, thicknesses of the CFN were measured using the Vernier caliper at all sites.

The LCNC was extremely thin and had various origins. The branching pattern of the LCNC was classified according to its origin: type 1a, from the lateral side of the CFN; type $1 \mathrm{~b}$, from the medial side of the CFN; type 2 , 


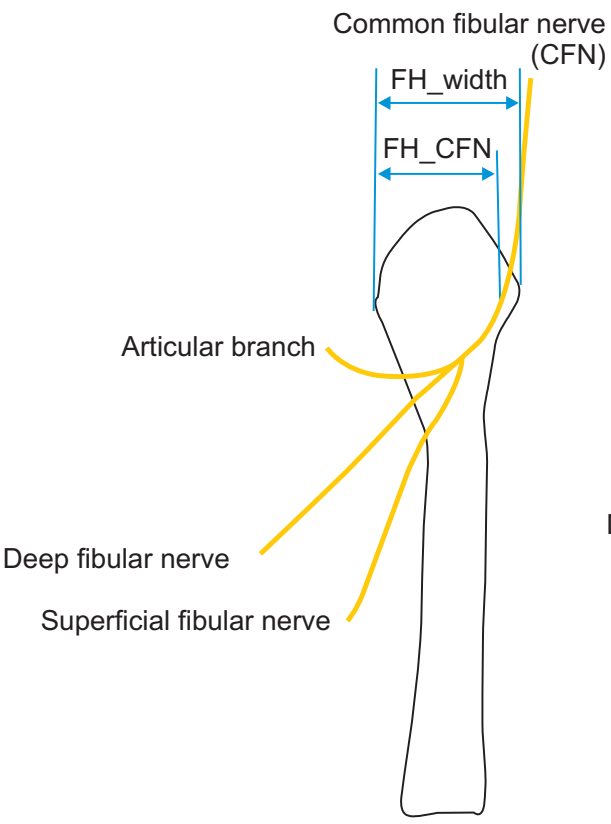

Cross type

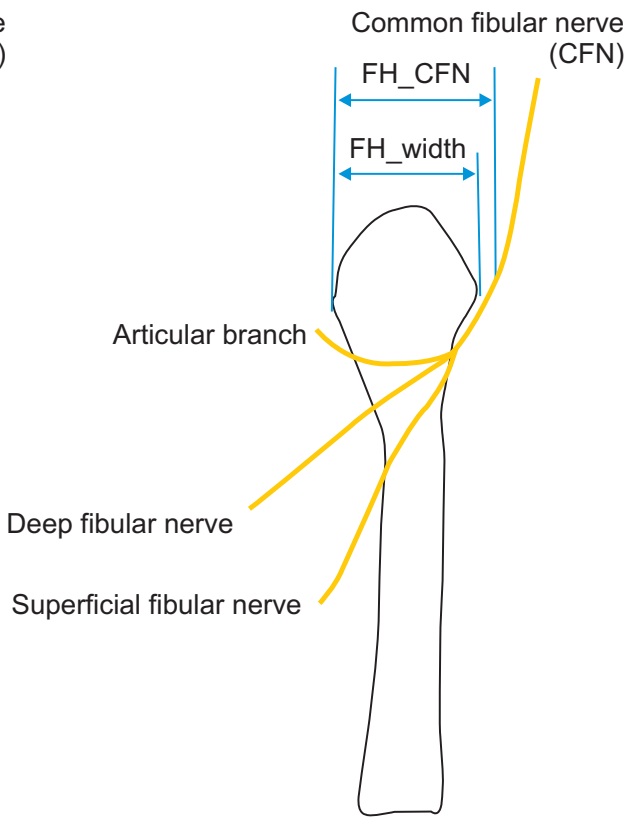

Posterior type
Fig. 2. Relationship between the fibular head and common fibular nerve. FH_width, the fibular head width; FH_CFN, the distance between the common fibular nerve and the anterior margin of fibular head.

Table 1. Common course of the fibular nerve at the fibular head and common fibular nerve angle at $5 \mathrm{~cm}$ proximal to the fibular head

\begin{tabular}{|lccc|}
\hline & Cross type (n=15) & Posterior type (n=6) & p-value \\
\hline Age $(\mathrm{yr})$ & $81.0(84-89)$ & $78.5(69-85)$ & 0.569 \\
\hline FH_CFN (mm) & $20.0(14.9-24.5)$ & $23.0(19.0-27.9)$ & 0.080 \\
\hline FH_width (mm) & $26.8(20.8-31.5)$ & $21.5(12.0-27.9)$ & $0.023^{*}$ \\
\hline Ratio (FH_CFN/FH_width) & $0.83(0.59-0.88)$ & $1.04(1.00-1.58)$ & $0.000^{*}$ \\
\hline Angle $\left(^{\circ}\right)$ & $13.0(8.0-20.5)$ & $11.0(9.0-17.0)$ & 0.340 \\
\hline
\end{tabular}

Values were presented as median (minimum-maximum).

Cross type, case in which the common fibular nerve course was across the fibular head; Posterior type, case in which the common fibular nerve course was posterior to the fibular head; FH_CFN, distance between the common fibular nerve and anterior margin of the fibular head; $\mathrm{FH}_{-}$width, fibular head width; Angle, angle between the common fibular nerve and tibial nerve $5 \mathrm{~cm}$ proximal to the fibular head.

${ }^{*} \mathrm{p}<0.05$.

from the LSCN; and type 3, from the lateral side of the CFN and LSCN. The distance between the tip of the FH and branching point of the LCNC was measured using the Vernier caliper.

\section{RESULTS}

The median thigh length, that is, the distance from the ischial tuberosity to the $\mathrm{FH}$, was $335 \mathrm{~mm}$ (range, 279-385 $\mathrm{mm})$.

In Table 1, various factors for each cross and posterior type were summarized. At the mid-FH level, the common course of the fibular nerve was observed across the FH in 15 cases and posterior to the FH in 6 . In the cross type of the CFN around the FH, median values of the FH_CFN and FH_width were 20.0 and $26.8 \mathrm{~mm}$, respectively, and the ratio of FH_CFN to FH_width (FH_CFN/FH_width) was 0.83 . In the posterior type, median values of the FH_CFN and FH_width were 23.0 and $21.5 \mathrm{~mm}$, respectively, and the ratio of FH_CFN to FH_width (FH_CFN/ FH_width) was 1.04. FH_width and ratio (FH_CFN/FH_ width) had statistically significant results, with p-values of 0.023 and 0.000 , respectively.

Next, median values of the angle between the CFN and 
tibial nerve in the $5 \mathrm{~cm}$ proximal position of the $\mathrm{FH}$ were $13.0^{\circ}$ in the cross type and $11.0^{\circ}$ in the posterior type. The CFN with a greater angle was driving closer to the FH. Therefore, the cross type had a greater angle than the posterior type, but without statistical significance.

Median values of the thickness of the CFN were measured for each sites. The thickness of the CFN was still 9.8 $\mathrm{mm}$ before it split with the tibial nerve in the popliteal fossa (superior margin). The thickness was reduced to 4.5 $\mathrm{mm}$ at the $7 \mathrm{~cm}$ proximal to the $\mathrm{FH}$, and then $5.0 \mathrm{~mm}$ and $5.0 \mathrm{~mm}$. However, it increased to $6.2 \mathrm{~mm}$ at the $\mathrm{FH}$ and to $8.6 \mathrm{~mm}$ at the fibular neck (Fig. 3).

Table 2 shows various branching patterns of the LCNC. Type la was observed in 5 cases, type $1 \mathrm{~b}$ in 5 , type 2 in 10 , and type 3 in 1 . In addition, distances between the branching point of the LCNC and FH were measured in

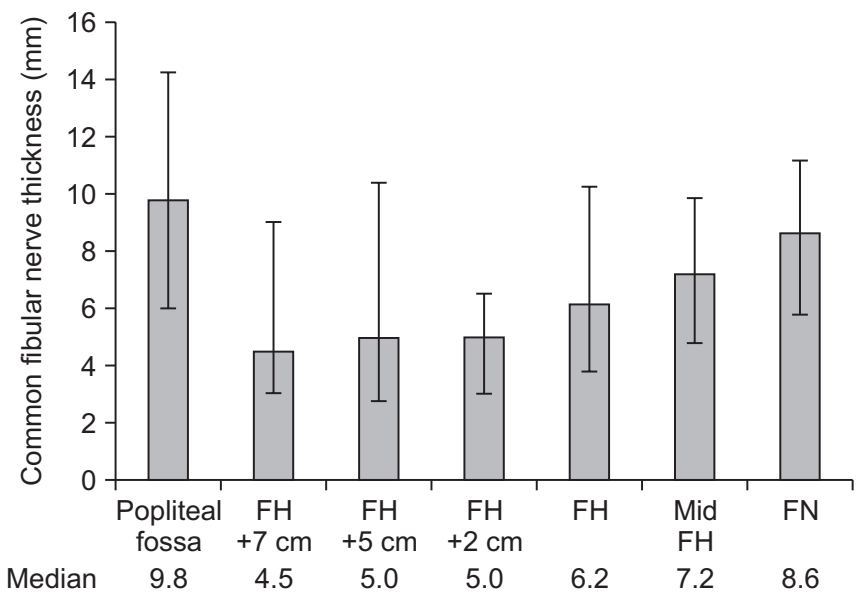

Fig. 3. Graph of the common fibular nerve thickness at each site. Values were presented as median. Ranges (minimum-maximum) were indicated by the error bars. $\mathrm{FH}+\mathrm{n} \mathrm{cm}, \mathrm{n} \mathrm{cm}$ above the fibular head; $\mathrm{FH}$, fibular head; Mid FH, midpoint of the fibular head; FN, fibular neck.
Table 2. The LCNC was branched at $30.0 \mathrm{~mm}$ distal to the FH for type 1a, $10.1 \mathrm{~mm}$ for type $1 \mathrm{~b}, 24.0 \mathrm{~mm}$ for type 2, and $13.0 \mathrm{~mm}$ for type 3 as the median value.

Actual dissection images and simple representation of the branching pattern in each type are classified, and the yellow line represents the LCNC (Fig. 4).

\section{DISCUSSION}

According to Van den Bergh et al. [1], anatomically, the relatively little epineurium supporting the tissue around the FH, superficial placement of the CFN, thin and weak nerves, and ossification of the peroneus longus muscle origin may cause compression of the CFN. The proximal bifurcation of the CFN is prone to injury, especially during arthroscopic knee surgeries. In most cases, there is very little fat around the $\mathrm{FH}$, and the CFN runs just around the $\mathrm{FH}$, attached completely to the $\mathrm{FH}$, where it is susceptible to compression. Moreover, the CFN is thinner than the tibial nerve. In many cases in the present study, the CFN crossed the FH instead of running posterior to it. In patients who are bedridden for a prolonged time, damage to the CFN may occur if the knee is held at a lateral rotation position for a long duration.

The course of the CFN in the present study can provide information for the accurate stimulation of the nerve around the FH. Many textbooks recommend stimulation sites around the $\mathrm{FH}$ as just below and lateral to the $\mathrm{FH}$ or a few centimeters distal to the FH $[5,6]$. As per Table 1, the CFN crosses the FH but can also pass posterior to the FH. Therefore, when an examiner stimulates this nerve at the FH, they should stimulate a relatively wide area and determine a point where a good amplitude, that is, the maximum response from pure CFN, can be observed.

The thickness of the CFN varied according to the level,

Table 2. Origin type of the LCNC and distance between the tip of the fibular head and the branching point of the LCNC

\begin{tabular}{llcl}
\hline & \multicolumn{1}{c}{ LCNC origin } & Number of cases & Distance (mm) \\
\hline Type 1a & Lateral margin of the CFN & 5 & $30.0(20.0-59.2)$ \\
\hline Type 1b & Medial margin of the CFN & 5 & $10.1(-6.0-48.0)$ \\
Type 2 & LSCN & 10 & $24.0(-22.0-35.5)$ \\
\hline Type 3 & Lateral margin of the CFN; LSCN & 1 & 13.0 \\
\hline
\end{tabular}

Values were presented as median (minimum-maximum). Minus value indicates that the LCNC courses was medial to the origin nerve.

Distance, distance between the fibular head and the lateral cutaneous nerve branching point; LCNC, lateral cutaneous nerve of the calf; CFN, common fibular nerve; LSCN, lateral sural cutaneous nerve. 


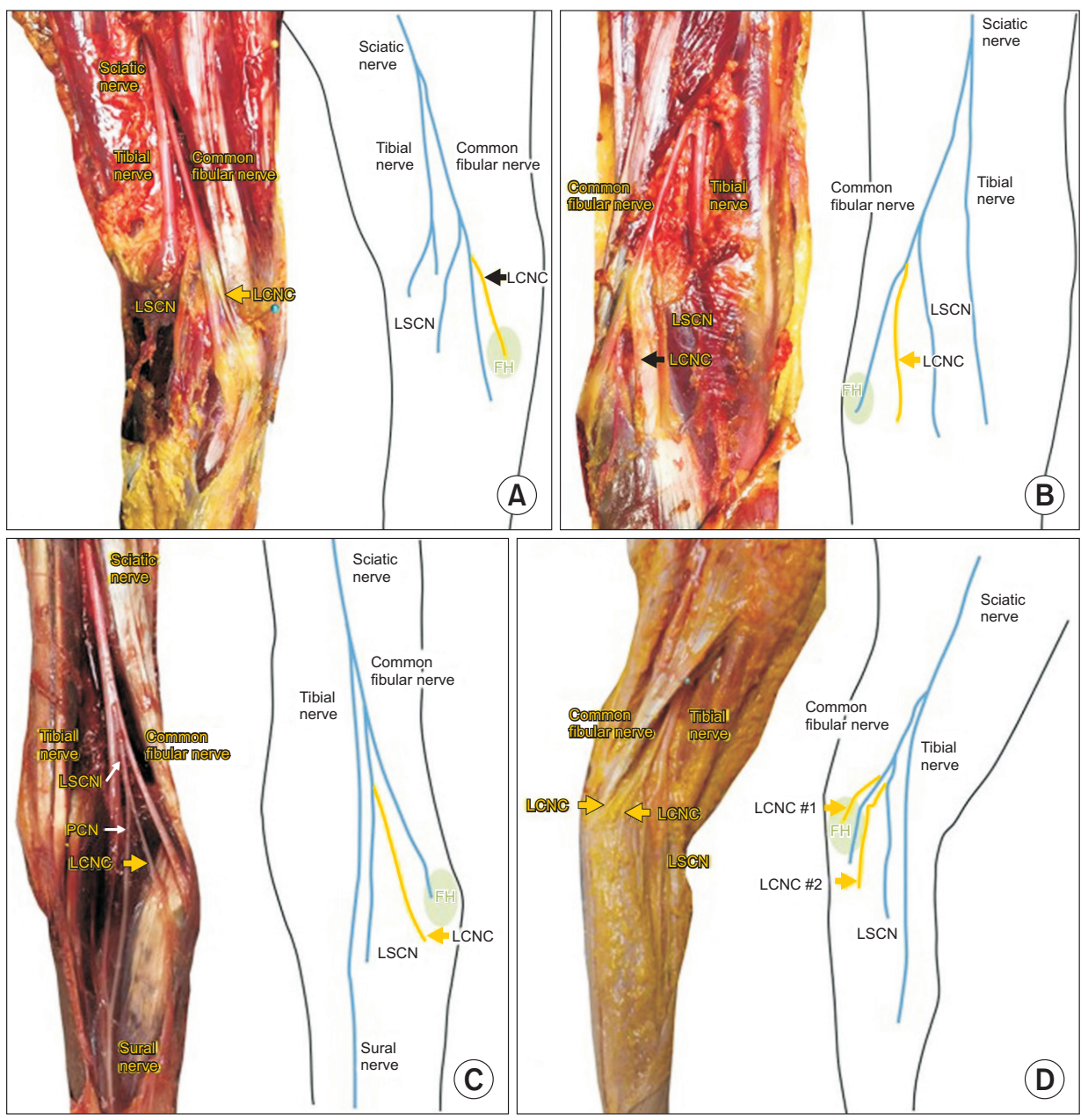

Fig. 4. Branching pattern of the lateral cutaneous nerve of the calf (LCNC). (A) LCNC originated from the lateral margin of the common fibular nerve. (B) LCNC originated from the medial margin of the common fibular nerve. (C) LCNC originated from the lateral sural cutaneous nerve (LSCN). (D) Two LCNC originated from the lateral margin of the common fibular nerve and the LSCN. The yellow line corresponds to the LCNC. FH, fibular head. as shown in Fig. 3. As the CFN moves towards the FH, space became smaller and its cross-section took on an oval shape. In addition, most cadavers in this study were from patients who had been bedridden for an extended duration; thus, compression of the fibular nerve may have occurred. Lateral knee compression (as can occur during bed rest with lateral rotation of the leg) and malnutrition are commonly comorbid with bed rest or prolonged surgery. Because malnutrition leads to weight loss, it may act as a confounder when considering positioning as the cause of fibular nerve neurapraxia [3].

In a case study performed by Hackam and Zwimpfer [7], the patient showed symptoms of tingling, numbness, and pain in the right calf. The Tinel sign was positive at the distal popliteal fossa. On physical examination, motor weakness and sensory loss related to the CFN were not observed, and electrophysiologic examination of the CFN revealed normal results. In surgery, the LCNC was entrapped in the biceps femoris tendon, and the patient was treated with surgical decompression. Injury to the LCNC is uncommon, but this nerve can be compressed near the FH owing to the thickening of structures such as the fibrous band, indicating the entrapment symptom [4]. Such cases may be confused with common fibular neuropathy or L5 radiculopathy, but there is limited research on this nerve. In the dissection performed in the present study, the LCNC was organized from the various origins as the lateral and medial sides of the CFN and LSCN.

A study assessed variations in the medial sural cutaneous nerve, LSCN, and peroneal communicating nerve through the dissection of 50 lower limbs [8]. In that study, the LSCN was absent in $18 \%$ of cases and arose directly from the CFN in 66\%. According to Pyun and Kwon [9] and Ramakrishnan et al. [10], the origin of the sural nerve is also very diverse. The subtypes are determined according to the presence of the peroneal communicating nerve, LSCN, and medial sural cutaneous nerve. In other words, the presence of these nerves varies among indi- 
viduals; therefore, the origin of the LCNC is also variable. Examination of the LCNC is difficult due to the presence of several variations. The nerve conduction method described by Lee and DeLisa [5] recommends that the examiner should stimulate the nerve by placing the cathode $2 \mathrm{~cm}$ posterior and $4 \mathrm{~cm}$ proximal to the $\mathrm{FH}$ and placing the E1 electrode $12 \mathrm{~cm}$ distal to the cathode in the direction of the calcaneus. In all types, distances between the branching point of the LCNC and FH were variable, from $13.0 \mathrm{~mm}(1.3 \mathrm{~cm})$ to $30.0 \mathrm{~mm}(3 \mathrm{~cm})$. If the conduction study does not present good results, the examiner can try stimulating a point closer to the FH.

The study of Campagnolo et al. [11] described an orthodromic and antidromic conduction technique for the stimulation of the LCNC. The method described by Lee and DeLisa [5] was used, and 64 lower limbs were enrolled. Considering the small size of the LCNC, small sensory nerve action potential (SNAP) was acceptable. In 21 limbs, conduction was possible using both antidromic and orthodromic techniques, and SNAP was unobtainable in all 4 limbs. The antidromic method was more responsive than the orthodromic method, and the latter method was shown to have less diagnostic significance. As the LCNC has a small size and many variations, both antidromic and orthodromic methods need to be used in studies.

The present study has a few limitations. First, the number of dissected limbs was small and too limited to assess the CFN around the knee. More cadaveric studies can help to generalize the anatomical course and branching patterns of the CFN. Second, most fresh cadavers had been in a long disease period and bedridden; thus, there was a high likelihood of prior compression of the CFN. Additionally, it would also be helpful to compare our study results with sonography results from living people.

In conclusion, an anatomical correlation between the FH and CFN at the mid-FH level could be related with the development of common fibular neuropathy around the knee level because of stretching or compression on the FH.

There were various branching patterns of the cutaneous nerves of the CFN around the popliteal fossa, especially the LCNC, which would be related to mononeuropathy of the LCNC, although the condition is rare.

\section{CONFLICT OF INTEREST}

No potential conflict of interest relevant to this article was reported.

\section{ACKNOWLEDGMENTS}

This study was supported by a grant from Korea University (No. K1824851).

\section{AUTHOR CONTRIBUTION}

Conceptualization: Kim DH. Methodology: Kim DH, Kim GY, Ryou CH, Kim D. Formal analysis: Kim DH, Kim GY. Project administration: Kim DH. Visualization: Kim DH, Kim GY. Writing - original draft: Kim GY. Writing review and editing: Kim DH, Kim KH, Rhyu IJ. Approval of final manuscript: all authors.

\section{REFERENCES}

1. Van den Bergh FR, Vanhoenacker FM, De Smet E, Huysse W, Verstraete KL. Peroneal nerve: normal anatomy and pathologic findings on routine MRI of the knee. Insights Imaging 2013;4:287-99.

2. Poage C, Roth C, Scott B. Peroneal nerve palsy: evaluation and management. J Am Acad Orthop Surg 2016; 24:1-10

3. Baima J, Krivickas L. Evaluation and treatment of peroneal neuropathy. Curr Rev Musculoskelet Med 2008; 1:147-53.

4. Khalil NM, Nicotra A, Kaplan C, O'Neill KS. Entrapment of the lateral cutaneous nerve of the calf. BMJ Case Rep 2013;2013:bcr2013008847.

5. Lee HJ, DeLisa JA. Manual of nerve conduction study and surface anatomy for needle electromyography. 4th ed. Philadelphia: Lippincott Williams \& Wilkins; 2004.

6. Dumitru D. Electrodiagnostic medicine. Philadelphia: Hanley \& Belfus; 1995.

7. Hackam DG, Zwimpfer TJ. Congenital entrapment of the lateral cutaneous nerve of the calf presenting as a personal sensory neuropathy. Can J Neurol Sci 1998; 25:168-70.

8. Kavyashree AN, Subhash LP, Asha KR, Bindurani MK. Study of variations in medial sural cutaneous nerve, 
lateral sural cutaneous nerve and peroneal communicating nerve. J Int Med Dent 2014;1:10-8.

9. Pyun SB, Kwon HK. The effect of anatomical variation of the sural nerve on nerve conduction studies. Am J Phys Med Rehabil 2008;87:438-42.

10. Ramakrishnan PK, Henry BM, Vikse J, Roy J, Saganiak
$\mathrm{K}$, Mizia E, et al. Anatomical variations of the formation and course of the sural nerve: a systematic review and meta-analysis. Ann Anat 2015;202:36-44.

11. Campagnolo DI, Romello MA, Park YI, Foye PM, Delisa JA. Technique for studying conduction in the lateral cutaneous nerve of calf. Muscle Nerve 2000;23:1277-9. 